\title{
Measurement of kinetic constant of protein binding using microfluidics and particle diffusometry
}

\author{
Hui $\mathrm{Ma}^{1}$, Steven T. Wereley ${ }^{2}$, Jacqueline C. Linnes ${ }^{1 *}$, Tamara L. Kinzer-Ursem ${ }^{1 \dagger}$ \\ ${ }^{1}$ Weldon School of Biomedical Engineering, Purdue University, West Lafayette, Indiana, 47907, USA \\ ${ }^{2}$ Department of Mechanical Engineering and Birck Nanotechnology Center, Purdue University, West \\ Lafayette, Indiana 47907, USA \\ * jlinnes@purdue.edu \\ $\dagger$ tursem@purdue.edu
}

\begin{abstract}
Protein-protein interaction is widely used in biological science and biomedical engineering research Moreira et al. (2007). Accurate measurement of binding kinetics is essential for understanding protein-protein interactions. Current gold standard assays, such as surface plasmon resonance (SPR), bio-layer interferometry (BLI) and quartz crystal microbalance (QCM), can generate precise and real-time kinetics data. However, these methods usually require expensive instruments housed in core facilities and high-level expertise, which is not convenient for most labs to implement. We developed a new method based on microfluidics and particle diffusometry (PD) to measure protein binding kinetics, which only needs very general lab equipment including a fluorescent microscope to take photos, a syringe pump to inject solutions, capillary tubing, a simple chip made on a glass slide and a computer to process images. To measure the binding rate of a protein pair, both proteins are conjugated with beads of different sizes, respectively. The bead solutions are diluted to appropriate concentrations and injected into a Y-junction channel by a syringe pump. In the microchannel, the two kinds of beads will meet at the interface and bind due to surface protein interactions. Therefore, the size of the beads in solution gradually increases and the Brownian motion will be less and less drastic until the reaction is saturated. Taking photos recording this dynamic process, the apparent change in size of the beads can be measured by particle diffusometry and used for extracting binding kinetics. Particle diffusometry is a correlation-based and non-intrusive optical detection method to analyze properties of fluid and particle such as viscosity, temperature and particle diameterChamarthy et al. (2009); Clayton et al. (2016, 2017b a); Hohreiter et al. (2002). It was initially developed to determine errors caused by thermal noise in particle image velocimetry (PIV). PD always analyzes image pairs. A single image of a particle laden flow is first used to do auto-correlation, correlating with itself, which will generate a high and sharp peak. Then it is cross-correlated with a successive image with a known time interval $\Delta t$. Because particles slightly deviate away from the initial positions after time $\Delta t$, due to Brownian motion, the correlation peak is lower and broader than that of auto-correlation. Auto- and cross-correlation peaks are fit into Gaussian function to find peak widths, by which the particle size can be computed as long as the viscosity and temperature do not change. Processing the image sets of the protein-conjugated particles' binding process, we acquire the relation of particle size and time, which can be used to solve protein binding kinetics. An equation of protein interaction and particle volume is derived to work out association rate from particle diameter data acquired by PD. In this study, we measured streptavidin-biotin binding rate. Streptavidin is conjugated with $20 \mathrm{~nm}$ beads and biotin is immobilized onto $200 \mathrm{~nm}$ beads. Proteins on the two kinds of beads bind rapidly after mixing in the main channel. It is necessary to choose a narrow area at the interface of particle streams that diffusion does not limit the reaction. Since the liquid is flowing, there is both Brownian motion and advection in particle images. We used EDPIV, a software package developed by Prof. Steven Wereley's lab, to measure advection velocity. When doing PD analysis, images are shifted following the PIV data to catch up with the flow. The photos are taken at the center layer in the middle of the channel, where there is no velocity gradient. Measuring a series of photo sets along the main channel at several points with known distances to each other, the relation of complex bead size and time can be acquired. Solving for the association constant, the measured value is $1.74 \times 10^{7} \mathrm{M}^{-1} \mathrm{~s}^{-1}$, which is close to that of current gold standard assays. This novel PD-based method is accurate and requires only general lab facilities, making protein binding kinetics measurements accessible and practical for biological and biomedical labs.
\end{abstract}




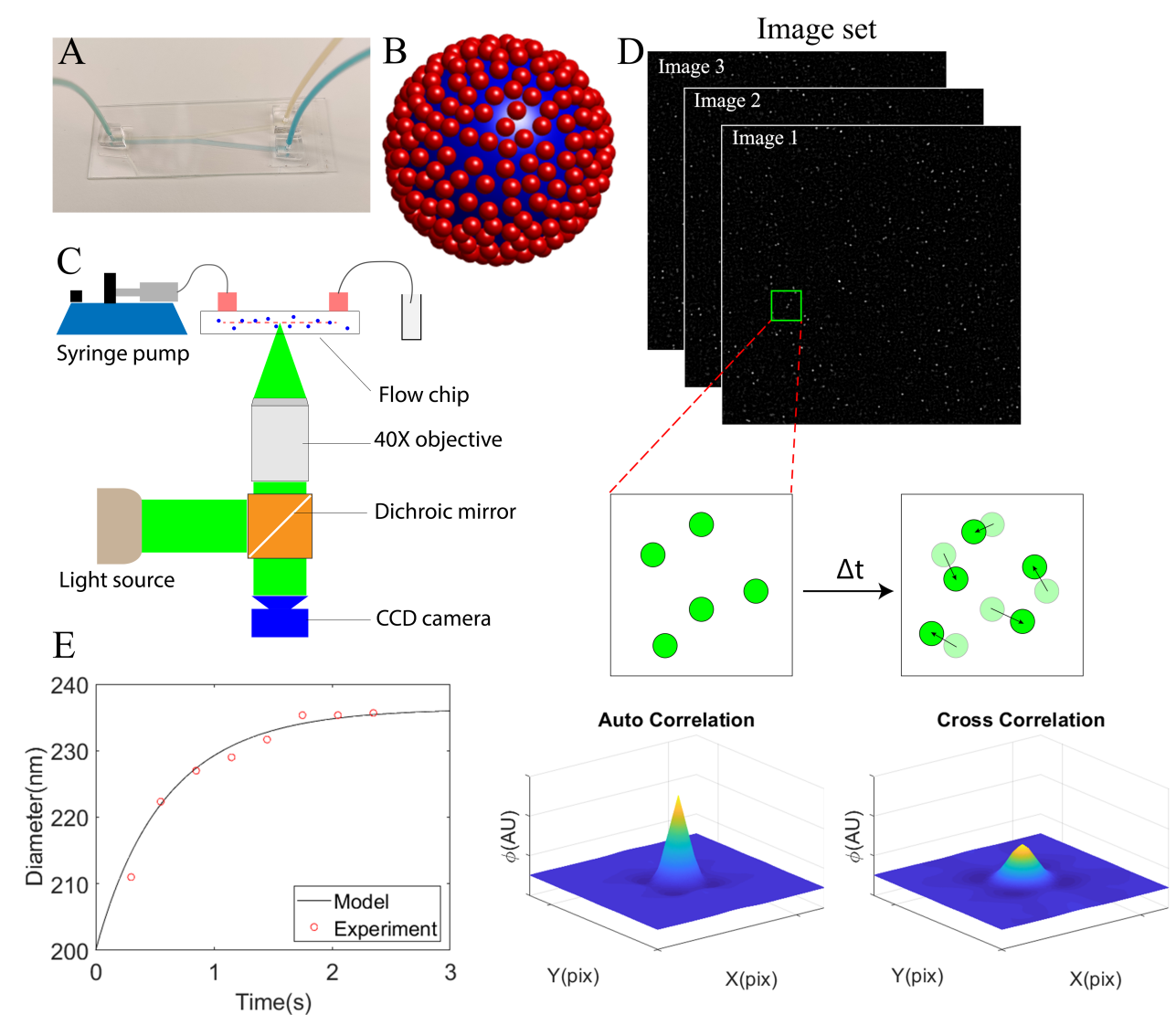

Figure 1: A. Y-junchtion channel fabricated from glass, PDMS, and pressure sensitive adhesive. Solutions are injected through the two arms and meet in the main channel. B. MATLAB model of 20nm streptavidinbeads bound to $200 \mathrm{~nm}$ biotinylated bead. Particle size increases due to binding can be measured by PD. C. Experiment setup. Solutions are injected by a syringe pump. A fluorescent microscope is used to take photos. D. PD mechanism. An interrogation window correlates with itself (auto-correlation), generating a high and sharp peak. An interrogation window correlated with the next successive image (cross-correlation), producing a lower and border peak due to the particles' Brownian motion. PD can be employed to calculate particle size. E. Measured experiment data of bead size with the binding of streptavidin and biotin beads over time.

\section{References}

Chamarthy P, Garimella SV, and Wereley ST (2009) Non-intrusive temperature measurement using microscale visualization techniques. Experiments in fluids 47:159-170

Clayton KN, Berglund GD, Linnes JC, Kinzer-Ursem TL, and Wereley ST (2017a) Dna microviscosity characterization with particle diffusometry for downstream dna detection applications. Analytical chemistry 89:13334-13341

Clayton KN, Lee D, Wereley ST, and Kinzer-Ursem TL (2017b) Measuring biotherapeutic viscosity and degradation on-chip with particle diffusometry. Lab on a chip 17:4148-4159

Clayton KN, Salameh JW, Wereley ST, and Kinzer-Ursem TL (2016) Physical characterization of nanoparticle size and surface modification using particle scattering diffusometry. Biomicrofluidics 10:054107

Hohreiter V, Wereley S, Olsen M, and Chung J (2002) Cross-correlation analysis for temperature measurement. Measurement science and technology 13:1072

Moreira IS, Fernandes PA, and Ramos MJ (2007) Hot spots-a review of the protein-protein interface determinant amino-acid residues. Proteins: Structure, Function, and Bioinformatics 68:803-812 